\title{
A Brainstem-like Modulation Approach for Gait Transition in a Quadruped Robot
}

\author{
Vítor Matos, Cristina P. Santos and Carla M.A. Pinto
}

\begin{abstract}
The ability to traverse a wide variety of terrains while walking is basically a requirement for performing useful tasks in our human centric world.

In this article, we propose a bio-inspired robotic controller able to generate locomotion and to easily switch between different type of gaits. In order to improve the robot stability and response while locomoting, we adjust both the duty factor and the interlimb phase relationships, according to the velocities.

We extend previous work, by applying nonlinear oscillators to generate the rhythmic locomotor movements for a quadruped robot, similarly to the biological counterparts. The generated trajectories are modulated by a drive signal, that modifies the oscillator frequency, amplitude and the coupling parameters among the oscillators, proportionally to the drive signal strength. By increasing the drive signal, locomotion can be elicited and velocity increased while switching to the appropriate gaits. This drive signal can be specified according to sensory information or set a priori.

The implementation of the central pattern generator network and the activity modulation layer is shown in simulation and in an AIBO robot.
\end{abstract}

\section{INTRODUCTION}

Animals use different gaits to suit the walking speed, using statically stable gaits for slower speeds and dynamically stable gaits for faster speeds. Under some conditions of motion, a certain gait is more adequate for reasons that are related to stability, speed, energy efficiency, terrain properties, mobility or structure of the animal [1]. At all walking speeds the onset of swing in a foreleg occurs just after the onset of stance in the ipsilateral hind leg. Thus, phase relationships between diagonal limbs gradually reach synchronization with the increase of speed (decrease of duty factor) [1], i.e., both the duty factor and the interlimb phase relationships should be changed according to the speed increase. This improves the stability of the locomotion because the support of the body smoothly changes from three points support (walk) to two points support (trot) [2], [3], [4].

This gait switching problem has already been addressed in robotics [5], [6], [7], [3], [2]. However, the obtained results are somehow limited for several reasons: whether the robots were specifically designed for the control strategy, or the strategy was restricted to a small number of legs or only one gait was explored.

Vítor Matos and Cristina Santos are with Industrial Electronics Department, School of Engineering, University of Minho, 4800058 Guimaraes, Portugal vmatos@dei.uminho.pt, cristina@dei.uminho.pt

Carla Pinto is with Instituto Superior de Engenharia do Porto, Centro de Matemática da Universidade do Porto, Rua Dr António Bernardino de Almeida, 431, 4200-072 Porto, Portugal
In this work, we propose a controller architecture for robotic quadruped locomotion. This architecture must be able to generate different motor behaviours for a robotic quadruped, namely locomotion initiation, smooth gait switching according to speed change and to stop locomotion. Further, the architecture is only feed with a unique signal, a modulatory drive, which elicits one of these motor behaviours. This signal can be set a priori or according to sensory information.

The architecture is bio-inspired in the vertebrate biological motor systems [1], [8], [9], [10], and is structured in functional hierarchical layers according to their level of abstraction. Similar ideas are presented in [11].

The lower level addresses the role of the spinal cord and generates the motor patterns by networks of Central Pattern Generators (CPGs). Based on previous work [12], [13], [14], we apply (oscillator-based) differential equations to model a network of four coupled limb-CPGs. This network produces coordinated rhythms of motor activity, i.e. the correct pattern for locomotion. These systems are solved using numerical integration and sent to the lower level PIDs of the joints.

Unidirectional communication from layer two to one happens through specification of the CPG parameters that control layer one.

The second layer models very basically the brainstem command centers for initiating, regulating and stopping CPGs activity and therefore initiate a walking gait, switch among gaits and stop the locomotion. This layer receives a modulatory signal which strength is mapped onto different sets of the CPG parameters, and hence result in the different motor behaviours.

In this sense, this contribution represents a step forward in the attempt to achieve flexible, adaptive goal-directed locomotion. The proposed controller is simpler when compared to other solutions [15], enables to elicit behavior switching, and improves the stability and the response of the robot during its locomotion, for the various velocities.

The proposed system is implemented and tested in both, a simulated environment and a real robot. Results demonstrate that stability does not change abruptly as without this gradual interlimb phase adjustment, but rather decreases approximately linearly when velocity increases. Further, behavior switching is smoothly and easily achieved by simple modification of a single drive signal. Results also show how behavior switching can be elicited from sensory information. 


\section{NEURAL STRUCTURES FOR LOCOMOTION IN} VERTEBRATES

Intensive biological research has provided a description of the neural basis involved in locomotion generation [8], adaptation [16] and goal-directed locomotion [9].

Basically, networks of Central Pattern generators (CPGs), located at the spinal level, generate the motor pattern. These networks of CPGs control much of the timing, pattern, amplitude and rhythm of muscle activation [17].

The initiation and regulation of the activity of these networks is determined from the brainstem locomotor command systems [18]. Different stimulation strengths induce gait transitions, such that low stimulation gives rise to the slow walking gait and progressively greater strength will induce changes from walking to trotting to galloping. Meaning that, in quadrupeds, the speed of locomotion increases and there are changes in interlimb coordination.

This organization appears to be conserved throughout vertebrate evolution [9].

The nervous system contains several different motor behaviours, stored as motor programs, designed to solve a variety of motor tasks, from simple to complex. Once activated and timed, these motor behaviours will produce different types of motor tasks.

\section{A. Gait transition}

During locomotion, quadruped walking animals have to usually move their legs in a manner that provides the suitable forward force at a minimal energy expenditure while maintaining their equilibrium. This coordinated cyclic manner of lifting and placing the legs on the ground, called a gait, is important for equilibrium stability and the step cycle sequence is typical for vertebrates: left forelimb (LF), right hindlimb $(\mathrm{RH})$, right forelimb (RF), and left hindlimb (LH).

In general, the number of step cycles per second increases as the speed of locomotion increases [1]. This corresponds to a reduction in the step cycle duration almost exclusively due to a shortening of the stance phase (limb in contact with the ground), whereas the swing phase (no ground contact) is kept nearly constant.

Quadrupedal gaits are classified according to the duration of their stance phases [4], i.e. their duty factor values, and their relative phases. In general, the duty factor $\beta$ reduces as the speed increases.

The considered crawl and trot gaits are symmetric gaits, meaning that the two legs of the same girdle are 0.5 out of phase. These gaits are singular (two or more legs are simultaneously lifted or placed during a stride) and regular (all the legs have the same duty factor).

Herein, we assume that at all walking speeds the onset of swing in a foreleg occurs just after the onset of stance in the ipsilateral hind leg [1]. In order to achieve this, we use the wave gait rule: the gait phase $\left(\varphi_{3}\right)$ follows the value of the duty factor $(\beta)$. The use of this rule improves the stability of the locomotion [2], [4], [3]. We calculate the stability margin [2] which decreases approximately linearly with the velocity increase (see results).

\section{LOCOMOTOR MODEL}

We propose an architecture inspired in the vertebrate biological systems [9],structured in two functional hierarchical layers with different levels of abstraction. The lower level addresses the role of the spinal cord and generates the motor patterns by networks of CPGs. The second layer models very basically the brainstem command centers for initiating, regulating and stopping CPGs activity and therefore initiate a walking gait, switch among gaits and stop the locomotion.

\section{A. Pattern generation}

Considering an implementation of a controller for quadruped locomotion there are several features that are relevant. Stability and smoothness enable more natural movements, reduce the risk of the robot loosing stability while walking and the risk of damage. Also, simple parameter changes in the controller should be able to generate different types of motor behaviours. This switch should be easily elicited and according to sensory information. It must also be able to independently control the swing and stance durations, since to change the gait we need to change the stance phase duration while keeping the swing duration constant. Least but not last, interlimb coordination should be automatically adjusted such that the lag between the diagonal limbs shortens with increasing speed (walk to trot).

The dynamical systems approach [15], [19] applies well to model CPGs for robotic controllers. Trajectories are generated on-line and in real time with a low computational cost. Further, these systems allow smooth online modulation of the generated trajectories with respect to their amplitude, frequency (for speed change) and offsets by simple, small parameter changes, while keeping the general features of the original movements. These systems also provide for robustness against small perturbations because of existence of globally stable attractors (limit cycle behaviors) and as such allow for integration of sensory feedback and ensure robust control of the movements in time-varying environments [12], [13].

1) Limb-CPGs: The rhythmic locomotor movements for each hip limb, $i$, are generated by the following Hopf oscillator

$$
\begin{aligned}
\dot{x}_{i} & =\alpha\left(\mu-r_{i}^{2}\right) x_{i}-\omega z_{i}, \\
\dot{z}_{i} & =\alpha\left(\mu-r_{i}^{2}\right) z_{i}+\omega x_{i},
\end{aligned}
$$

where $r_{i}=\sqrt{\left(x_{i}\right)^{2}+z_{i}^{2}}$, amplitude of the oscillations are given by $A=\sqrt{\mu}, \omega$ specifies the oscillations frequency (in $\operatorname{rad} s^{-1}$ ) and relaxation to the limit cycle is given by $\frac{1}{2 \beta \mu}$.

This oscillator contains an Hopf bifurcation from a stable fixed point at $x_{i}=0$ (when $\mu<0$ ) to a structurally stable, harmonic limit cycle, for $\mu>0$.

This oscillator facilitates the smooth modulation of the generated trajectories according to changes in the amplitude, goal and frequency parameters. This is interesting for trajectory generation in a robot [12] and for modelling the CPGs. Both are able to generate motor patterns without sensory feedback and without any rhythmic inputs, when activated 


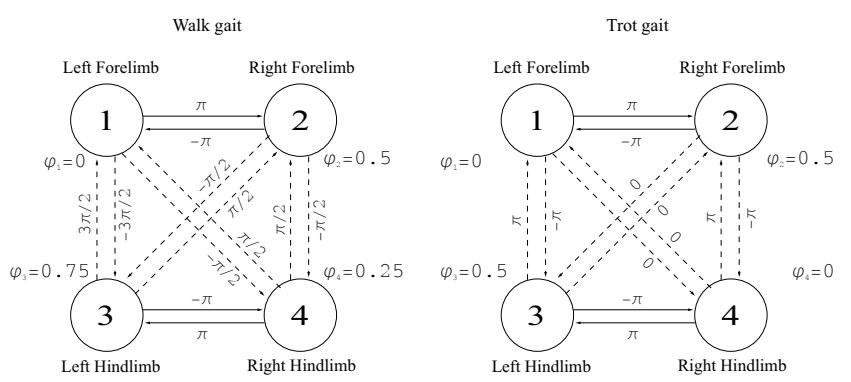

(a) Each leg lags one quarter of a (b) Diagonal legs move in synchrony cycle, in the sequence: Left Fore, while contralateral legs move in strict Right Hind, Right Fore, Left Hind alternation.

Fig. 1. Relative gait phase for each limb and relative phases $\left(\theta_{i}^{j}\right)$ between the oscillators, for the walk and trot gaits. Solid arrows represent the alternating relation, that never changes for alternating (or symmetric) gaits.

by simple commands that somehow encode their rhythmic activation, frequency and amplitude.

The following expression for $\omega_{i}$ (3) allows an independent control of speed of the ascending and descending phases of the rhythmic signal [20], meaning an independent control of the stance $\omega_{\mathrm{st}}$ and the swing durations $\omega_{\mathrm{sw}}$.

$$
\omega=\frac{\omega_{\mathrm{st}}}{\mathrm{e}^{-a z_{i}}+1}+\frac{\omega_{\mathrm{sw}}}{\mathrm{e}^{a z_{i}}+1}
$$

2) Interlimb coordination: In order to achieve interlimb coordination, the locomotor system should be regarded as composed by four interconnected limb-CPGs coupled as follows

$$
\left[\begin{array}{c}
\dot{x}_{i} \\
\dot{z}_{i}
\end{array}\right]=\left[\begin{array}{cc}
\alpha\left(\mu-r_{i}^{2}\right) & -\omega \\
\omega & \alpha\left(\mu-r_{i}^{2}\right)
\end{array}\right]\left[\begin{array}{c}
x_{i} \\
z_{i}
\end{array}\right]+\sum_{j \neq i} \mathbf{R}\left(\theta_{i}^{j}\right)\left[\begin{array}{c}
x_{j} \\
z_{j}
\end{array}\right]
$$

The linear terms are rotated onto each other by the rotation matrix $\mathbf{R}\left(\theta_{i}^{j}\right)$, where $\theta_{i}^{j}$ is the required relative phase among the $i$ and $j$ hip oscillators to perform the gait (we exploit the fact that $\mathbf{R}(\theta)=\mathbf{R}^{-1}(-\theta)$ ).

The final result is a network of oscillators with controlled phase relationships, able to generate more complex, synchronized behavior such as locomotion. Due to the properties of this type of coupling among oscillators, the generated trajectories are stable and smooth and thus potentially useful for trajectory generation in a robot.

The relative phases are specified according to the walk (Fig. 1(a)) and the trot gaits (Fig. 1(b)), in which contralateral legs always move in strict alternation. By re-arranging (4) according to these relative phases and in terms of the gait phase $\varphi_{3}$, the equations for each hip joint become

$$
\begin{aligned}
& \dot{x_{1}}=f_{\mathrm{x}, 1}\left(x_{1}, z_{1}\right)-x_{2}-\mathbf{c} x_{3}-\mathbf{s} z_{3}-\mathbf{c} x_{4}+\mathbf{s} z_{4} \\
& \dot{z_{1}}=f_{\mathrm{z}, 1}\left(x_{1}, z_{1}\right)-z_{2}+\mathbf{s} x_{3}+\mathbf{c} z_{3}-\mathbf{s} x_{4}-\mathbf{c} z_{4} \\
& \dot{x_{2}}=f_{\mathrm{x}, 2}\left(x_{2}, z_{2}\right)-x_{1}-\mathbf{c} x_{3}+\mathbf{s} z_{3}+\mathbf{c} x_{4}-\mathbf{s} z_{4} \\
& \dot{z_{2}}=f_{\mathrm{z}, 2}\left(x_{2}, z_{2}\right)-z_{1}-\mathbf{s} x_{3}-\mathbf{c} z_{3}+\mathbf{s} x_{4}+\mathbf{c} z_{4} \\
& \dot{x_{3}}=f_{\mathrm{x}, 3}\left(x_{3}, z_{3}\right)-x_{4}+\mathbf{c} x_{1}+\mathbf{s} z_{1}-\mathbf{c} x_{2}-\mathbf{s} z_{2} \\
& \dot{z_{3}}=f_{\mathrm{z}, 3}\left(x_{3}, z_{3}\right)-z_{4}-\mathbf{s} x_{1}+\mathbf{c} z_{1}+\mathbf{s} x_{2}-\mathbf{c} z_{2} \\
& \dot{x_{4}}=f_{\mathrm{x}, 4}\left(x_{4}, z_{4}\right)-x_{3}-\mathbf{c} x_{1}-\mathbf{s} z_{1}+\mathbf{c} x_{2}+\mathbf{s} z_{2} \\
& \dot{z_{4}}=f_{\mathrm{z}, 4}\left(x_{4}, z_{4}\right)-z_{3}+\mathbf{s} x_{1}-\mathbf{c} z_{1}-\mathbf{s} x_{2}+\mathbf{c} z_{2}
\end{aligned}
$$

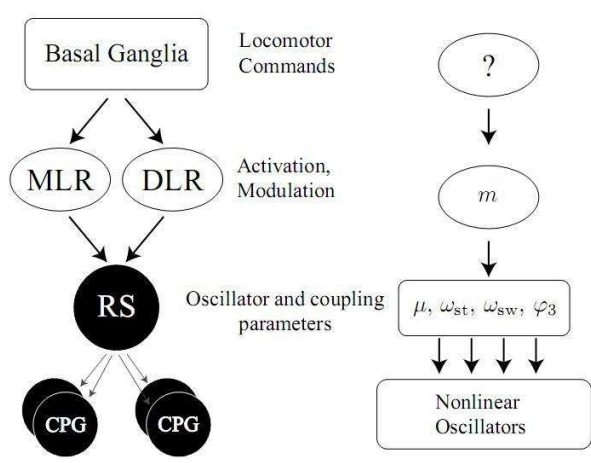

Fig. 2. The lower level generators receive excitatory and modulatory signals (parameters). Commands from higher centers and/or information from sensorial centers are mapped onto these parameters.

where $f_{\mathrm{x}, i}\left(x_{i}, z_{i}\right)$ is given by $(1,2), \mathbf{s}$ and $\mathbf{c}$ represent the $\sin \left(2 \pi \varphi_{3}\right)$ and $\cos \left(2 \pi \varphi_{3}\right)$ functions, respectively.

The $x_{i}$ variable generates trajectories for the $i$ hip swing joint of the robot limbs. These trajectories are sent online for the lower level PID controllers of each joint. The knee joints are controlled as simple as possible: the knee is flexed to a fixed angle during swing phase, and extended to a fixed angle during the stance phase.

This constitutes the lower level of the architecture that receives from the upper level the required $\mathrm{CPG}$ parameters to modulate the generated trajectories.

\section{B. Brainstem activation of motor programs}

In our model, a given modulatory drive signal, $m$, regulates the activity of the network of limb-CPGs. The strength of this drive initiates, stops and switches among gaits, by changing the required CPGs parameters. The tuned CPG parameters are the amplitude, $\mu$; the gait phase $\varphi_{3}$; and the frequencies, $\omega_{\mathrm{sw}}$ and $\omega_{\mathrm{st}}$, of the swing and stance phases, respectively. Moreover, interlimb coordination is automatically shifted according to the modulatory drive strength. Herein, both the range and the thresholds for the modulatory drive were chosen arbitrarily.

A parallel between a simplified biological architecture and the proposed one is presented in fig. 2 .

1) Initiating/stopping locomotion: Qualitatively, by modifying on the fly the $\mu$ parameter, the system $(1,2)$ switches between a stable fixed point at $x_{i}=0(\mu<0)$ and a purely rhythmic movement $(\mu>0)$. Hence, the $\mu$ parameter controls whether or not there are oscillations generated by the limbCPG. Bellow a lower threshold, $m_{\text {low }}=0.2$, the oscillators are shut down and the robot rests.

The parameter $\mu$ is set as a piecewise linear function of the drive (Fig. 3), such that

$$
\mu=f_{1}(m)= \begin{cases}-A^{2} & , m<m_{\text {low }} \\ A^{2} & , m \geq m_{\text {low }}\end{cases}
$$

2) Duty factor modulation: As the modulatory drive increases in strength, the duty factor, $\beta$, linearly decreases from 0.89 to the crawl gait to $\beta=0.5$ for the trot gait. The 


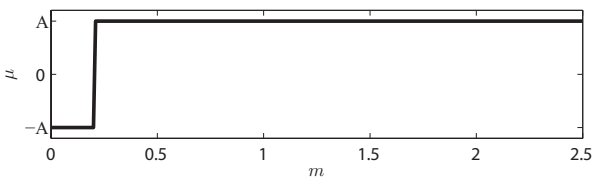

Fig. 3. Oscillator's parameter $\mu=f_{1}(m)$. For a modulatory drive greater than 0.2 the oscillators are activated.

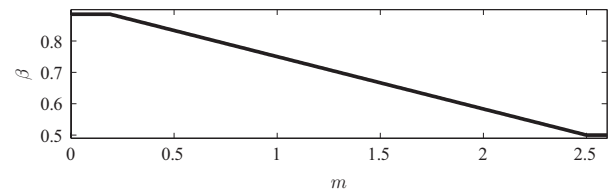

Fig. 4. Oscillator's parameter $\beta=f_{2}(m)$. The duty factor $(\beta)$ decreases linearly with the increase of modulatory signal, until reaching 0.5 .

duty factor is mathematically defined as a piecewise linear function of the modulatory drive (Fig. 4)

$\beta=f_{2}(m)=\left\{\begin{array}{lr}0.89 & , m<m_{\text {low }} \\ -0.1667 m+0.9167 & , m_{\text {low }} \leq m<2.5 \\ 0.5 & , m \geq 2.5\end{array}\right.$

The stance phase frequency, $\omega_{\mathrm{st}}$, is determined based on the constant swing frequency, $\omega_{\mathrm{sw}}$, and on the desired duty factor, $\beta$ as follows

$$
\omega_{\mathrm{st}}=\frac{1-\beta}{\beta} \omega_{\mathrm{sw}}
$$

3) Interlimb coordination modulation: Previously to $m=$ 1 , the interlimb phase relationship is that of a crawl gait $\left(\varphi_{3}=0.75\right)$. Afterwards, the crawl gait slowly transfers into a trot gait $\left(\varphi_{3}=0.5\right)$ due to the increase in the modulatory drive. This is achieved by using the wave gait rule for a quadruped: the gait phase $\varphi_{3}$ should follow the value of the duty factor $(\beta)$. It remains in $\varphi_{3}=0.5$ for values of the modulatory drive greater than 2.5 (Fig. 5).

$\varphi_{3}$ is mathematically defined as a piecewise linear function of the drive, $m$,

$\varphi_{3}=f_{3}(m)=\left\{\begin{array}{lr}0.75 & , m<1 \\ -0.1667 m+0.9167 & , 1 \leq m<2.5 \\ 0.5 & , m \geq 2.5\end{array}\right.$

\section{Behavior switching}

Different values of the drive lead to different behaviors, namely: locomotion initiation, speed change and consequent gait change, similarly to the biological counterparts [10]. Different behaviors correspond to different specifications of

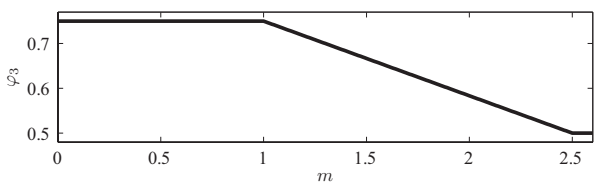

Fig. 5. Oscillator's parameter $\varphi_{3}=f_{3}(m)$. the set of the CPG parameters: amplitude, frequencies and gait phases.

Herein, the switch among these possible behaviors is defined a priori but could be defined according to time-varying sensory information, e.g., external stimuli [11]. For instance, in the experiments section, we present an experiment in which a touch in the back of the robot initiates the walking pattern. A continuous touch in its back elicits speed change and gradual transition and a touch in the head elicits stopping the locomotion.

\section{EXPERIMENTS}

To demonstrate the implementation of our model on a quadruped robotic system, we make several experiments. Experiments are done in a simulated AIBO quadruped robot using the Webots simulation software based on ODE, an open source physics engine.

The first experiment depicts comparison results between gait switching with and without interlimb phase adjustments.

In another experiment, switching between locomotion initiation, gait transition and locomotion stopping is done accordingly to a modulatory drive. Interlimb coordination is automatically adjusted to the duty factor value, accordingly to the modulatory drive strength. This drive is set a priori, in order to illustrate the easy switching among the possible different behaviors.

A final experiment is done in the real robot, by integrating a sensory pathway to determine the modulatory drive value, enabling the quadruped respond to real world stimuli and switch behaviours accordingly.

\section{A. Gradual vs Abrupt gait change}

In this experiment, we are interested in comparing the robot smoothness and performance in terms of stability margin when going from a statically stable gait (crawl gait) to a more faster trot gait, when interlimb phase relationships are and are not continuously adjusted. The crawl gait has a duty factor $\beta \geq 0.75$ and a gait phase $\varphi_{3}=0.75$. For faster speeds $(\beta<0.75)$, the walk of the robot is no more statically stable and the gait phase of walk is not adequate for the faster stepping frequency.

We expect a smoother locomotion and an improvement in the locomotion stability margin, when the interlimb phase relationships are changed according to the duty factor.

Two simulations are performed: one using a gradual transition between gaits, and another using an abrupt transition between gaits. In both the simulations, the robot starts in a walk gait and then slowly increases its stepping frequency by reducing the stance phase duration, until it reaches a duty factor of $\beta=0.5$. In both the simulations, the robot walks forward during the first $20 \mathrm{~s}$ in order to guarantee an initial stable crawl gait. We calculate the stability margin and measure the robot walking velocity.

In the first simulation, the robot gradually changes the gait phase accordingly to the duty factor (bottom panel of fig. 6), and reaches the trot gait when the duty factor and gait phase are 0.5 . 

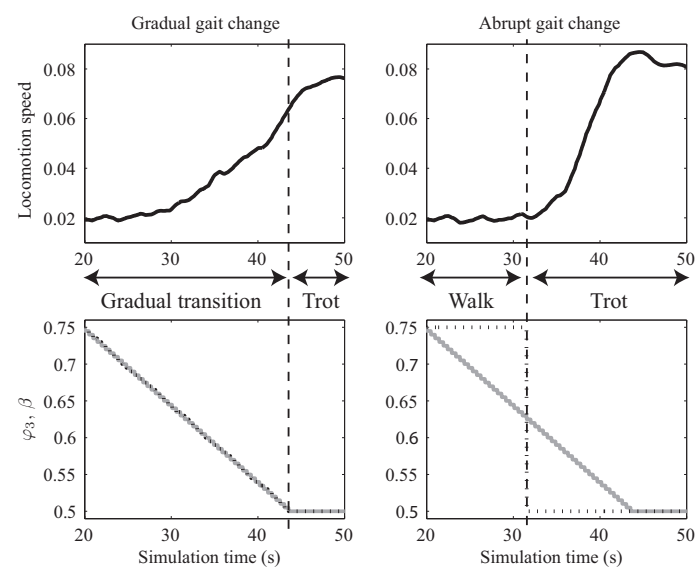

Fig. 6. Resulting locomotion speed (top) in the simulation when walking with the respective duty factor $\beta$ and gait phase $\varphi_{3}$ (bottom). When gradually changing the gait (left), the resulting locomotion speed increase is more smooth and more controllable than for the abrupt change of gait (right).
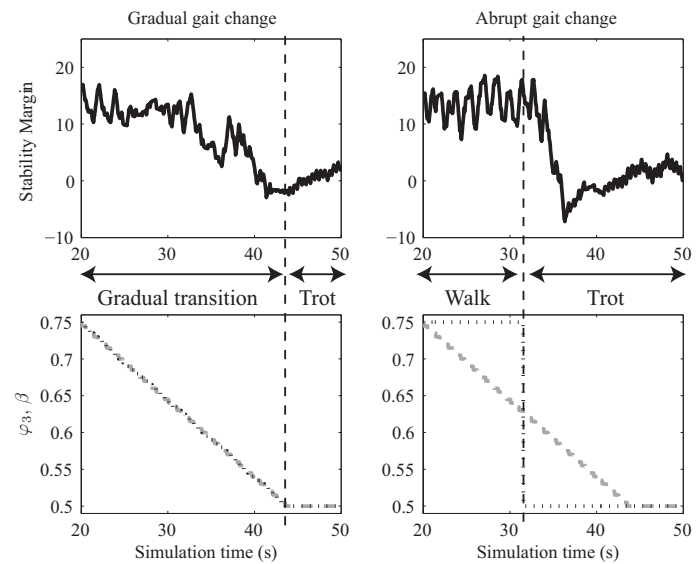

Fig. 7. Comparison of stability margin on gradual (left) and abrupt (right) transitions. The black solid line is the filtered stability margin from the simulation. The stability margin for the gradual gait change decreases slower than the abrupt gait change, which plummets right after the robot changes to trot.

In the second simulation, the robot only decreases the duty factor and the gait phase is changed when a duty factor of 0.625 is reached, half-way the duty factor of a walk and a trot.

Bottom panel of fig. 6 shows the locomotion speed evolution for both simulations. Velocity changes in a more smooth way when phase relationships vary according to the duty factor. This is relevant in a real robotics implementation, since the servos vary more gradually and are subjected to less strain.

Bottom panel of fig. 7 shows the stability margin evolution for both simulations. As expected, there is an improvement in the stability measure when phase relationships vary according to the duty factor. The stability margin decreases smoothly, i.e. gradually, as the gait is forced to a trot. In a real implementation this smoothness may minimize the need for strong reactions in order to recover equilibrium and/or stable locomotion.

\section{B. Locomotor behavior switching}

In this experiment, we are interested in showing the switching between different locomotor behaviors according to a modulatory drive, $m$, set a priori. Top panel of fig. 8 depicts the $m$ drive.

Initially, the drive is bellow $m_{\text {low }}$ and the robot rests.

Around $t=10.5 \mathrm{~s}$, the linearly increasing drive crosses the $m_{\text {low }}$ threshold and locomotion is initiated with a crawl gait (duty factor $\approx 0.88$ ).

The drive keeps linearly increasing but is bellow 1 for the next 15 seconds. Hence, the robot continues in the crawl gait, changing the duty factor but without phase relationships adjustments (second panel of fig. 8). At $t=25 \mathrm{~s}$, the robot keeps increasing its velocity but now gradually adjusting both the duty factor and the phase relationships. At $\mathrm{t}=40 \mathrm{~s}$, the drive is abruptly set to its maximum (2.5). The gait evolves to a trot gait, with the ipsilateral limbs in strict alternation and a duty factor of 0.5 . Servo joints are shown in the fourth panel.

After 10 seconds, the modulatory drive is set to zero, meaning that $\mu$ is set to a negative value (third panel) and the oscillators stop. Consequently, the locomotion is stopped. Bottom panel shows the locomotion speed evolution.

\section{Real robot}

The modulatory drive on this experiment is controlled by the capacitive touch sensors in the AIBO robot. This experiment is shown in the attached video. When the sensors in the back are touched, the modulatory drive is raised, and decreased when head sensor is touched. This means that switching between the possible locomotor behaviors is elicited by an external stimulus.

\section{CONCLUSION AND Future Works}

In this article we have extended the use of nonlinear oscillators for locomotion generation in legged robotic platforms, by implementing a mechanism for controlling the velocity and gait selection using one simple command.

We propose a second layer responsible for controlling the lower rhythmic generation layer, i.e., able to initiate/stop and modulate the rhythmic activity. This idea is inspired on the vertebrate locomotor network [18], that receives excitatory commands and convergence from higher centers, and in turn sends the respective activation signals for the pattern generators.

Specifically, the proposed layer controls the rhythmic generation of the locomotor movements, proportionally to the strength of the received drive signal. By increasing the drive signal, locomotion can be elicited and velocity increased while switching to the appropriate gaits.

We further discuss and implement a gradual shift between gaits to improve the stability and the response of the robot during its locomotion, for the various velocities.

Three experiments are presented. In the first experiment, we compare two simulations in order to show how much the walk of the robot improves when using a gradual transition between gaits. On the second experiment, we show how 


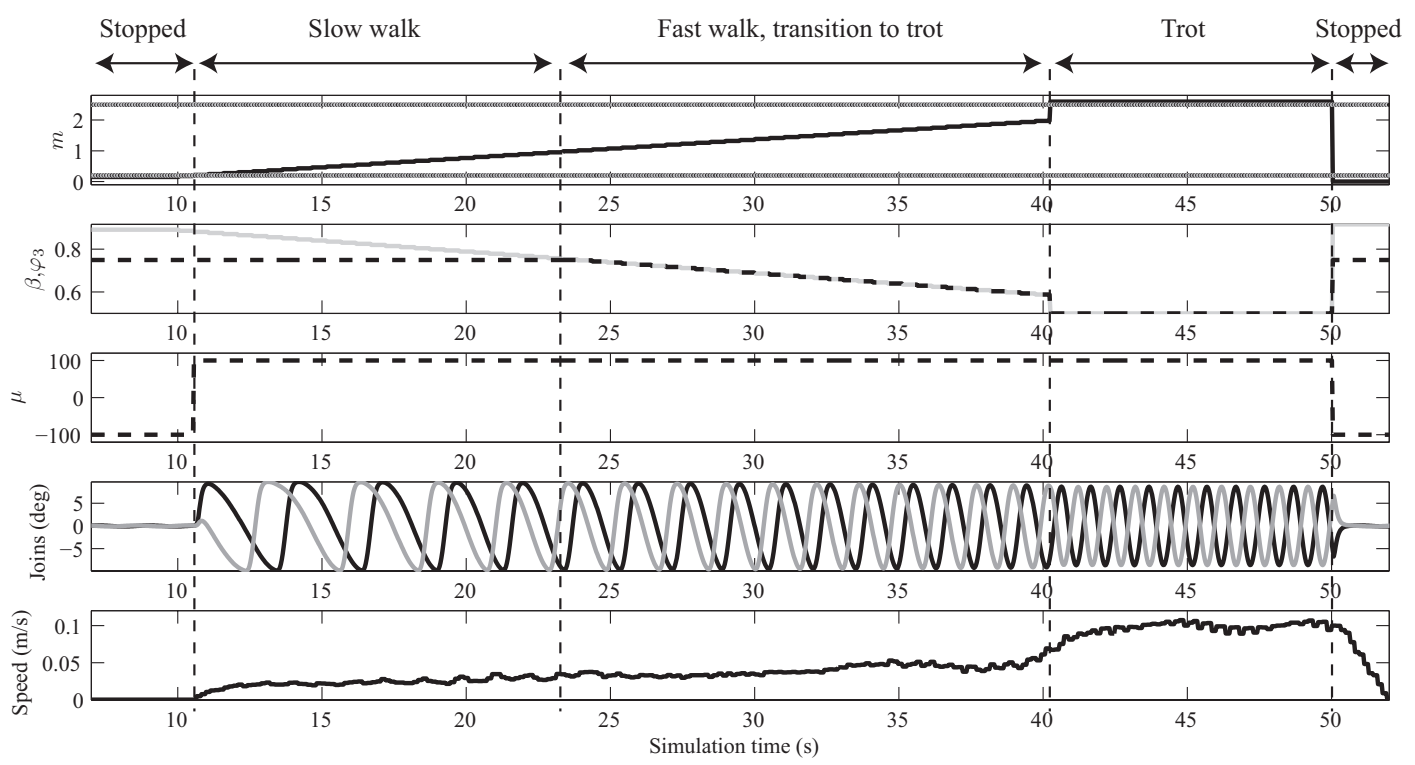

Fig. 8. The modulatory drive $(m)$ increases linearly in the $[1040]$ seconds interval, until it jumps to its maximum during the following 10 seconds (first panel). On the second panel, the solid black line is the duty factor $(\beta)$ and the grey solid line is the gait phase $\left(\varphi_{3}\right)$, which are encoded in the modulatory drive. The oscillator's parameter $\mu$ controls the state of the oscillators through the value of $m$ (third panel).The resulting trajectories of the robot hip joints are plotted in the fourth panel. The black line is the left forelimb joint and the grey line is the left hindlimb joint. The sudden increase of speed due the jump of modulatory drive can be seen in the fifth panel at 40 seconds.

the robot system behaves when a drive signal set a priori initiates, stops and switches between different gaits. In the third experiment, we implemented in the AIBO robot an interesting method for eliciting locomotion through stimuli, mimicking the sensory pathways in nature. The system's modulatory drive signal is increased and decreased by touching in the back and head of the robot, respectively.

For generating adaptive locomotion we are currently addressing accurate feet placement; predictive adjustments of locomotion including speed and/or step length control in advance and head stabilization for image acquisition. This work should also be integrated with our previous work for posture and balance control [13].

\section{ACKNOWLEDGMENTS}

The authors gratefully acknowledge Keir Pearson for all the discussions and help.

\section{REFERENCES}

[1] S. Grillner. Locomotion in vertebrates: central mechanisms and reflex interaction. Physiological Reviews, 55:247-304, 1975.

[2] Freyr Hardarson. Stability analysis and synthesis of statically balanced walking for quadruped robots. PhD thesis, KTH, 2002.

[3] K. Inagaki and H. Kobayashi. A gait transition for quadruped walking machine. Proceeding of the 1993 IEEE/RSJ International Conference on Intelligent Robots and Ssytems, 1993.

[4] R. B. Mcghee and A. A. Frank. On the stability properties of quadruped creeping gaits. Mathematical Biosciences, 3(1-2):331-351, August 1968.

[5] K. Yoneda and S. Hirose. Dynamic and static fusion gait of a quadruped walking vehicle. Brain Research Reviews, 9(3):7-15, 1991.

[6] A.Sano and J .Furusho. Static-dynamic transitional gait from crawl to pace. In ROBOMEC'92, volume B, pages 239-246, 1992.

[7] H. Yasa and M. Ito. An autonomous decentralized system with application to a gait pattern generator. Trans. of the Society of Instrument and Control Engineers, 26(12):180-187, 1989.
[8] M. MacKay-Lyons. Central pattern generation of locomotion: a review of the evidence. Phys Ther, 82(1):69-83, January 2002.

[9] S. Grillner, P. Wallna, K Saitoha, A. Kozlova, and B Robertsona. Neural bases of goal-directed locomotion in vertebratesan overview. Brain Research Reviews, 57(1):2-12, January 2008.

[10] S. Grillner, J. Hellgren, A. Ménard, K. Saitoh, and M. A. Wikström. Mechanisms for selection of basic motor programs-roles for the striatum and pallidum. Trends Neurosci, 28(7):364-370, July 2005.

[11] S. Degallier, L. Righetti, L. Natale, G. Metta F. Nori, and A. Ijspeert. A modular bio-inspired architecture for movement generation for the infant-like robot icub. In BioRob, 2008.

[12] Sarah Degallier, Cristina Santos, Ludovic Righetti, and Auke Ijspeert. Movement generation using dynamical systems: a humanoid robot performing a drumming task. In IEEE-RAS International Conference on Humanoid Robots, 2006.

[13] Luiz Castro, Cristina Santos, Miguel Oliveira, and Auke Ijspeert. Postural control on a quadruped robot using lateral tilt: A dynamical system approach. In EUROS, volume 44 of Springer Tracts in Advanced Robotics, pages 205-214. Springer, 2008.

[14] Cristina Santos, Miguel Oliveira, Ana Maria A.C. Rocha, and Lino Costa. Head motion stabilization during quadruped robot locomotion: Combining dynamical systems and a genetic algorithm. In IEEE International Conference on Robotics and Automation, 2009.

[15] Hiroshi Kimura, Yasuhiro Fukuoka, and Avis H. Cohen. Adaptive dynamic walking of a quadruped robot on natural ground based on biological concepts. Int. Journal of Robotics Research, 26(5):475490, 2007.

[16] G. Keir Pearson. Generating the walking gait: role of sensory feedback. Progress in Brain Research, 143:123-129, 2004.

[17] O. Kiehn. Locomotor circuits in the mammalian spinal cord. Annu Rev Neurosci, 29:279-306, 2006.

[18] M. Shimamura, S. Grillner, V. R. Edgerton, and Larry M. Jordan Brainstem and spinal cord mechanisms for the initiation of locomotion. In Neurobiological Basis of Human Locomotion Tokyo Japan Scientific Societies, 1991.

[19] A. Ijspeert, A. Crespi, D. Ryczko, and J.-M. Cabelguen. From swimming to walking with a salamander robot driven by a spinal cord model. Science, 315(5817):1416-1420, 2007.

[20] Ludovic Righetti and Auke Jan Ijspeert. Pattern generators with sensory feedback for the control of quadruped locomotion. In 2008 IEEE International Conference on Robotics and Automation, 2008. 